\title{
Simulation for Percutaneous Renal Access: Where Are We?
}

\author{
Yasser A. Noureldin, MD, MSc, PhD, ${ }^{1,2}$ and Sero Andonian, MD, MSc, FRCSC, FACS ${ }^{1}$
}

\begin{abstract}
Objectives: Percutaneous renal access (PCA) is a challenging step during percutaneous nephrolithotomy. The aim of this study is to review the literature for different types of simulators described for PCA.

Methods: Databases of Medline, Embase, Cochrane Library, OvidSP, and Google Scholar were systematically searched until May 2016. The studies were analyzed regarding the type of simulator (nonbiologic, biologic, live animal, and virtual reality [VR]), type of validity (face, content, construct, and predictive), cost-effectiveness, and whether these simulators have been used for training and/or assessment of PCA. In addition, the study looked at the educational impact of these simulators in terms of the transfer of PCA skills to the operating room. Results: Several bench, animal, and VR simulators for training in PCA were identified. Only few studies were found on assessment of PCA skills. Biological bench models used porcine or bovine kidneys wrapped within foam, silicone, chicken carcass, or full-thickness skin flap alone. Other biological models used additional subcutaneous fascia, muscle, or ribs. Nonbiological models used prototypes, including 3D printing. Only one study reported the use of anesthetized live pig for training. The PERC Mentor ${ }^{\mathrm{TM}}$ was the only VR simulator, which has been validated for training and assessment of PCA skills. However, none of these studies assessed the educational impact of PCA simulators. Furthermore, most of the studies did not address the validity and the cost of the simulator.

Conclusions: While several biological and nonbiological PCA models exist, there is paucity of literature regarding the validity and educational impact of these simulators. The PERC Mentor simulator is the sole validated simulator for training and assessment of PCA skills. However, it is expensive and there is little evidence of its educational impact. Therefore, more research is needed to validate the available simulators and assess their educational impact for urology trainees.
\end{abstract}

Keywords: percutaneous nephrolithotomy, computer simulation, clinical skills, outcome and process assessment

\section{Introduction}

$\mathbf{T}$ HERE IS NO DOUBT that percutaneous renal access (PCA) is a challenging step during percutaneous renal surgery. ${ }^{1,2}$ An appropriate PCA is considered an integral preliminary step during percutaneous nephrolithotomy (PCNL). This is due to the fact that the kidney is surrounded by important structures such as the colon, the liver (on the right side), the spleen (on the left side), the pleura, and the lungs (on both sides). In addition, the renal vasculature and renal pelvicaliceal orientation should be appropriately approached to minimize hemorrhagic complications. ${ }^{1}$ Therefore, inaccurate placement of the puncture needle can lead to injuries either to the kidney or to adjacent structures. ${ }^{3}$ While previous studies reported that percutaneous procedures are performed by $69.6 \%$ of practicing urologists, ${ }^{4}$ only $11 \%$ of them were found to obtain PCA themselves. ${ }^{3}$ Lack of training during urology training was cited as a cause. Other possible causes included personal preference, shortage of operating room (OR) time, financial considerations, or availability of better equipment in the radiology suite. ${ }^{5}$ Nevertheless, the need for independence regarding the timing and location of obtaining PCA pushed an increasing percentage of urologists to obtain the PCA themselves. ${ }^{1,6}$ In a retrospective single-center study, the outcomes of PCNL procedures were better whenever the PCA was obtained by the urologist rather than the interventional radiologist. ${ }^{3}$ However, achieving competency in PCA is quite difficult due to the steep learning curve and the fact that PCA is usually performed only once per PCNL procedure. $^{7,8}$ There are increasing concerns about patients' safety in light of decreased exposure to the OR and restricted training hours during the week. In addition, fluoroscopyguided PCA is the step that is accompanied with the most radiation exposure during PCNL. ${ }^{9}$ Therefore, simulators are

${ }^{1}$ Division of Urology, McGill University, Montréal, Québec, Canada.

${ }^{2}$ Urology Department, Benha University Hospital, Benha University, Benha, Egypt. 
needed for practicing an unlimited number of this critical step in a stress-free and radiation-free environment outside the OR, with flexibility regarding the time and place of practice. ${ }^{10,11}$ Different bench (nonbiological and biological), live animal, and virtual reality (VR) simulators have been introduced for training in PCA. Another important function of these VR simulators is that they give immediate formative feedback, which could be used for training and assessment of trainees in PCA skills. The aim of this study was to review the literature for the currently available simulators for training and assessment of PCA and address their cost-effectiveness and educational impact.

\section{Methods}

Databases of PubMed, Medline through OvidSP, Embase, Google scholar, and Cochrane library were systematically searched for articles written in the English language using the medical subject heading (MeSH) keywords: percutaneous nephrolithotomy, outcome and process assessment, clinical skills, and computer simulation. In addition, the following non-MeSH keywords were also searched: percutaneous access, skills, assessment, training, and virtual reality. This online search was conducted on June 15, 2016, without specifying the type of study and the year of publication. The search was broadened to include references from retrieved articles. All studies that described the development and/or validation of a simulator for training and assessment of PCA skills were included. To be consistent, all articles were reviewed by a single author (Y.A.N.). Review articles were excluded. The studies were analyzed regarding the type of simulator (bench, live animal, and VR), type of validity (face, content, construct, and predictive), cost-effectiveness, and whether the simulator has been used for training and/or assessment of PCA. In addition, the current review article examined the educational impact of simulators in terms of transfer of PCA skills from the simulator to the OR.

\section{Results}

Twenty studies on training and assessment of PCA were identified. Five review articles were excluded. Table 1 lists 20 studies in a chronological order of publication. It also summarizes their methodologies and conclusions. Table 2 summarizes the list of published simulators in addition to their type (biological, nonbiological, VR), cost, validity, training, assessment, and educational impact in terms of transferring PCA skills to the OR.

Several biological (porcine or bovine) and nonbiological, including VR, simulators for training in PCA were identified (Table 2). Most of the publications addressed validation of these simulators. Few studies were found on assessment of PCA skills. ${ }^{12,13}$ The biological bench models used porcine or bovine kidneys wrapped within foam, ${ }^{14}$ foam in a mannequin, ${ }^{15}$ silicone, ${ }^{16}$ chicken carcass, ${ }^{17,18}$ full-thickness skin flap, ${ }^{19,20}$ or additional subcutaneous fascia, muscle, ${ }^{21}$ and ribs. ${ }^{22}$ Other nonbiological bench models used prototyping or $3 \mathrm{D}$ printing. ${ }^{23-26}$ Only one study reported the use of anesthetized live pigs as module 1 of the Modular Training Scheme, which included renal puncture, tract dilatation using the Amplatz dilators, and orientation with the nephroscope in a live pig. ${ }^{27}$ The PERC Mentor ${ }^{\mathrm{TM}}$ (Simbionix, Cleveland, $\mathrm{OH})$ was the only VR simulator that has been validated for training and assessment of PCA skills. ${ }^{12,13,28-31}$ However, none of these studies assessed the educational impact of PCA simulators in terms of transferring PCA skills from the benchtop to the OR. Furthermore, most of these studies did not address the validity and the cost of these simulators.

\section{Training in PCA skills}

Training programs in Canada, Australia, Scotland, and United States have established competency as the target of training to meet the criteria of their certifying bodies. ${ }^{32}$ Competency is defined as "an observable ability of a health professional, integrating multiple components such as knowledge, skills, values, and attitudes." ${ }^{32}$ Recently, competency-based medical education (CBME) defined as "an outcome-based approach to the design, implementation, assessment, and evaluation of medical education programs using an organizing framework of competencies" has become the cornerstone of training, assessing, and promoting postgraduate trainees (PGTs). ${ }^{32}$ Therefore, different competency-based frameworks, such as the Outcome Project of the United States Accreditation Council for Graduate Medical Education, CanMEDS, and the Scottish Doctor, were described as the basis for CBME. ${ }^{33-35}$

Despite being the first-line option for managing large renal calculi, the indications for PCNL are expanding to include medium-sized and small renal calculi with recent modifications of the PCNL technique, such as mini-PCNL, ultraminiPCNL, and micro-PCNL. 6,36,37 However, PCA is still an integral step in all of these modifications and obtaining an appropriate PCA is mandatory for the PCNL procedure. ${ }^{1,3}$ In a study by de la Rosette and colleagues, ${ }^{38}$ learning PCA was responsible for the long learning curve of PCNL. Furthermore, a recent study by Noureldin et al. ${ }^{9}$ found that obtaining fluoroscopy-guided PCA is responsible for the higher radiation exposure during PCNL when compared with PCNLs performed in patients with preformed tracts. Thus, it is not surprising that the learning curve of PCNL is long, requiring 36-60 cases to achieve competency and $>100$ cases to achieve excellence. ${ }^{7,8,38}$ The First European Urolithiasis Society meeting during September 2011 confirmed the steep learning curve of PCA and identified two main etiologies that make training urology PGTs in PCA difficult: First, the complexity of obtaining a safe access to the kidney for lithotripsy. Second, fluoroscopic-guided access is being performed most of the time, thus the potential for injuring structures from the skin to the renal capsule. ${ }^{39}$ Therefore, it is mandatory for urology PGTs to receive appropriate PCNL training during their residency period to be familiar with this complex endourologic procedure. A recent report from the United Kingdom examined the exposure of urology PGTs to various urological procedures and noted that on average PGTs performed or assisted in only 19 PCNLs during their residency training period, while a wide variability among PGTs was noted with exposures to PCNL ranging from 0 to 125 procedures. ${ }^{40}$ Another survey from the United States showed that PGTs are comfortable with PCA after an average of $21.2 \pm 4.5$ procedures. ${ }^{5}$ Compared with urologists who were uncomfortable in performing PCA, urologists who were comfortable in performing PCA had performed significantly higher number of PCA procedures during their residency $(24.4 \pm 5.6 v s$ 10.6 $\pm 3.1 ; p=0.046) .^{5}$ Therefore, more than 24 PCA procedures were recommended to obtain the necessary 
PCA skills during the residency period. ${ }^{38}$ Thus, there should be a curriculum for training in PCNL during the urology residency programs. This curriculum should clearly describe the cornerstones for training in PCNL, define the learning curve, and determine surrogates in evaluating technical competency. In their proposal for minimal requirements of PCNL curriculum, de la Rosette and co-workers. ${ }^{38}$ defined the medical expertise in PCNL as theoretical and practical knowledge of the PCNL technique and its indications, and defined the technical expertise in PCNL as an appropriate access and lithotripsy in simple complicated and medium complicated PCNL cases under strict supervision. Previously, the stone-free rate and complications have been used as clinical surrogates for PCNL outcomes. Other parameters such as operative time, fluoroscopy time (FT), and estimated blood loss are also considered. ${ }^{41,42}$ However, recent reductions in residency work hours resulted in reduced access of PGTs to the OR. In addition, the fact that PCA is almost always performed only once per PCNL procedure necessitated the introduction of simulators for training PGTs outside the OR.

In this study, 14 simulators were identified for training in PCA; 12 dry laboratory bench models, 1 wet laboratory animal model, and 1 VR simulator (Table 2). Ex vivo animal models (biological bench models) have been used for PCNL training. These include porcine or bovine kidneys, with and without a part of the ureter, and with and without other parts of the body wall. The stones are introduced into the renal pelvis through a pyelotomy, which is then closed in a watertight manner, and the distal end of the ureter is tied over a ureteral catheter to give the opportunity to inject physiologic saline or contrast medium. ${ }^{16}$ Thus, PGTs can then train in PCA skills using either fluoroscopic or ultrasound guidance. These models offer tactile sensation that may be comparable with real life. However, preparation of these models consumes time and money. In addition, the PCA procedure can only be performed a limited number of times per model. Finally, there are ethical considerations in using animal models. All of these factors limit their availability for routine training.

VR simulators are software-dependent simulators creating an environment similar to the real clinical situation. They are user-friendly and enjoy several advantages: (1) the procedure could be repeated unlimited times in a stress-free and radiation-free environment, (2) they have multiple varieties of preset clinical scenarios with different levels of difficulty, and (3) they generate a performance report following completion of the task. Thus, objective assessment and monitoring of trainees' progress could be achieved. However, these simulators are expensive and may malfunction during practice since they are software dependent. ${ }^{43,44}$ The PERC Mentor (Simbionix) was the only VR simulator for PCNL training in normal and obese models using fluoroscopic guidance. $^{45}$ It gives the opportunity to practice PCA with various procedural steps such as (1) puncturing the pelvicaliceal system (PCS) using a $15-\mathrm{cm}$ needle for normal patients and $20-\mathrm{cm}$ needle for obese patients, (2) aspiration from the puncture needle using a virtual syringe producing a yellow color of urine, whenever the needle was introduced into the PCS and a red color of blood, whenever the needle hits a blood vessel causing vascular injury, (3) injecting a contrast medium through a virtual ureteral catheter, (4) monitoring the anatomy of the PCS, planning the puncture, and following the progress of the puncture needle into the PCS while the kidney is moving up and down with respiration, using biplanar VR $\mathrm{C}$-arm that could be controlled using a foot pedal, and (5) providing feedback on performance parameters such as procedure time, FT, number of attempts to puncture the PCS, number of vascular injuries, number of PCS perforations, number of rib collisions, and the amount of contrast medium used during antegrade and/or retrograde urography. ${ }^{1}$ In a study by Knudsen and colleagues, ${ }^{28}$ the PERC Mentor simulator was validated for training in PCA. ${ }^{29}$ However, it seems like VR simulators are underutilized in training of PGTs. This might be due to the lack of wide availability of these simulators due to their high cost. In a recent survey of 91 urology PGTs and 172 urologists from the United Kingdom, there was consensus on the lack of sufficient simulation-based urology training for common urological procedures such as cystoscopy (69\%-74\%), ureteroscopy (URS) (47\%-59\%), nephrectomy $(62 \%)$, transurethral resection of the prostate $(56 \%-65 \%)$, and percutaneous renal surgery $(73 \%-76 \%){ }^{46}$

\section{Assessment of PCA skills}

Clinical competence is the habitual and judicious use of communication, knowledge, technical skills, clinical reasoning, emotions, values, and reflection in daily practice for the benefit of the individual and community being served. ${ }^{47}$ Assessment of technical competency is an integral part of the whole assessment of clinical competency. This will not only give information about the fitness to practice, but also it provides constructive feedback for further learning and improvement. This is important as it has been confirmed that there is a significant negative correlation between the level of competency and patients' morbidity and mortality rates. ${ }^{48}$

Evaluation of competency involves assessment of skills, knowledge, beliefs, and attitudes in a quantitative and qualitative manner. ${ }^{49}$ Competency has been assessed using various assessment tools. These tools include generic and procedurespecific checklists, in-training evaluation reports, logbooks, direct observation of procedural skills, case-based discussion, outcome data on morbidity and mortality rates, peer assessment, multisource feedback, and evidence of participation in different clinical and nonclinical update courses. ${ }^{45,50-}$

${ }^{52}$ However, the lack of objectivity of these tools favors VR simulators, which enjoy objectivity in assessing technical skills. Objective assessment from VR simulators could be combined with validated subjective assessment tools, such as the global rating scale (GRS) and objective structured assessment of technical skills, to assess transfer of these skills to the OR. ${ }^{13,53}$ The GRS has been validated for assessing competency of trainees and specialists in real and simulated environments. ${ }^{53-55}$

Simulators used for assessment of PCA skills include human cadavers, live animals, and VR simulators to simulate human patients. ${ }^{56,57}$ In a recent study by Noureldin et al., ${ }^{12,13}$ the PERC Mentor simulator was incorporated into urology objective structured clinical examinations to assess PCA skills of PGTs. PGTs with previous PCA experience had performed the PCA with significantly shorter FT $(5.1 \pm 0.7 \mathrm{vs}$ $10 \pm 1.5 ; p=0.04)$, fewer infundibular injuries $(2 \pm 0.4 v s$ $7.4 \pm 1.5 ; p=0.004)$, fewer PCS perforations (4.5 $\pm 1.2 v s$ $11 \pm 1.7 ; p=0.01)$, and fewer attempts to puncture the PCS $(13 \pm 1.8$ vs $21 \pm 2.3 ; p=0.02)$ when compared with PGTs without previous PCA experience, respectively. ${ }^{12}$ Since the 


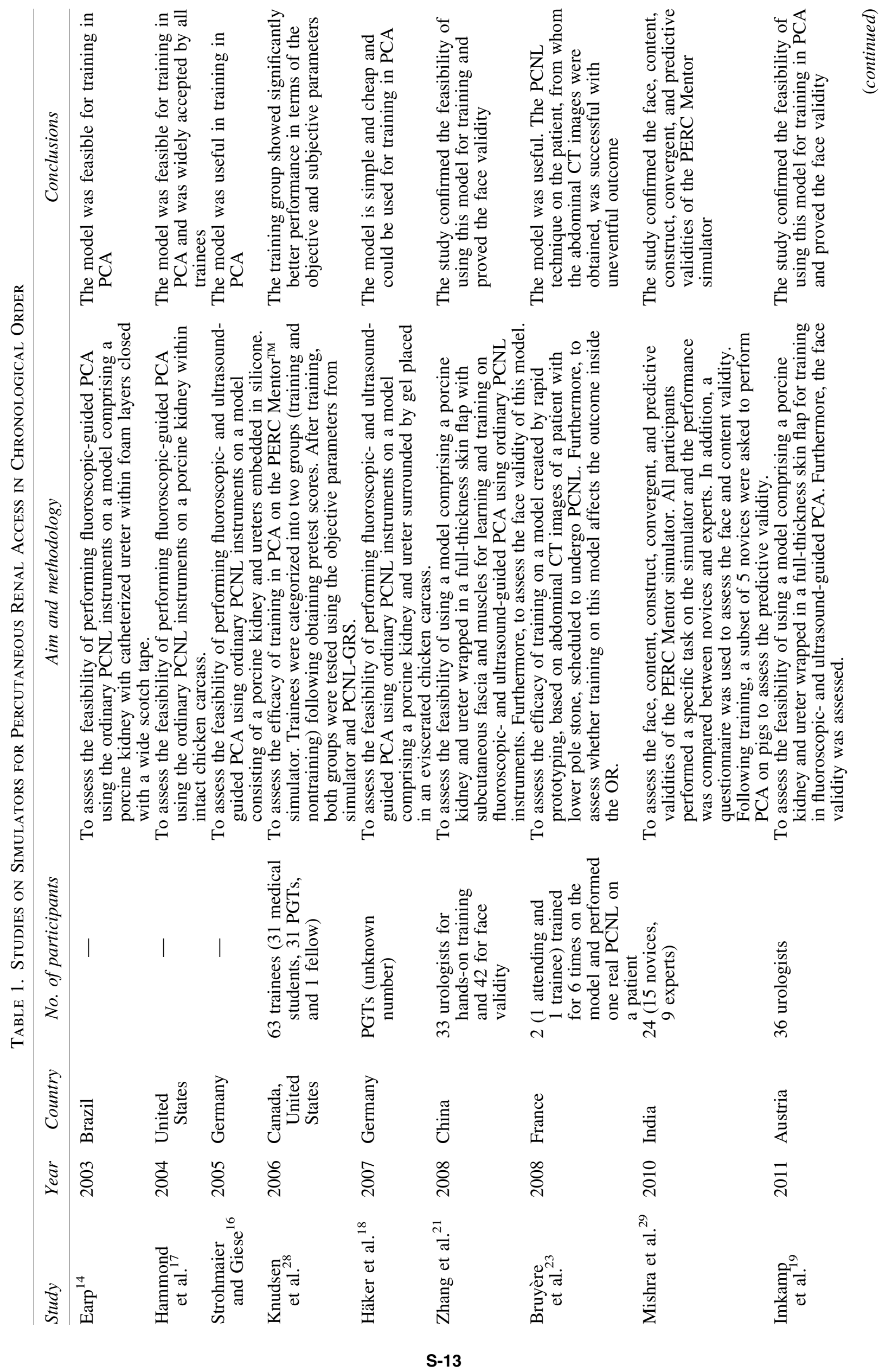




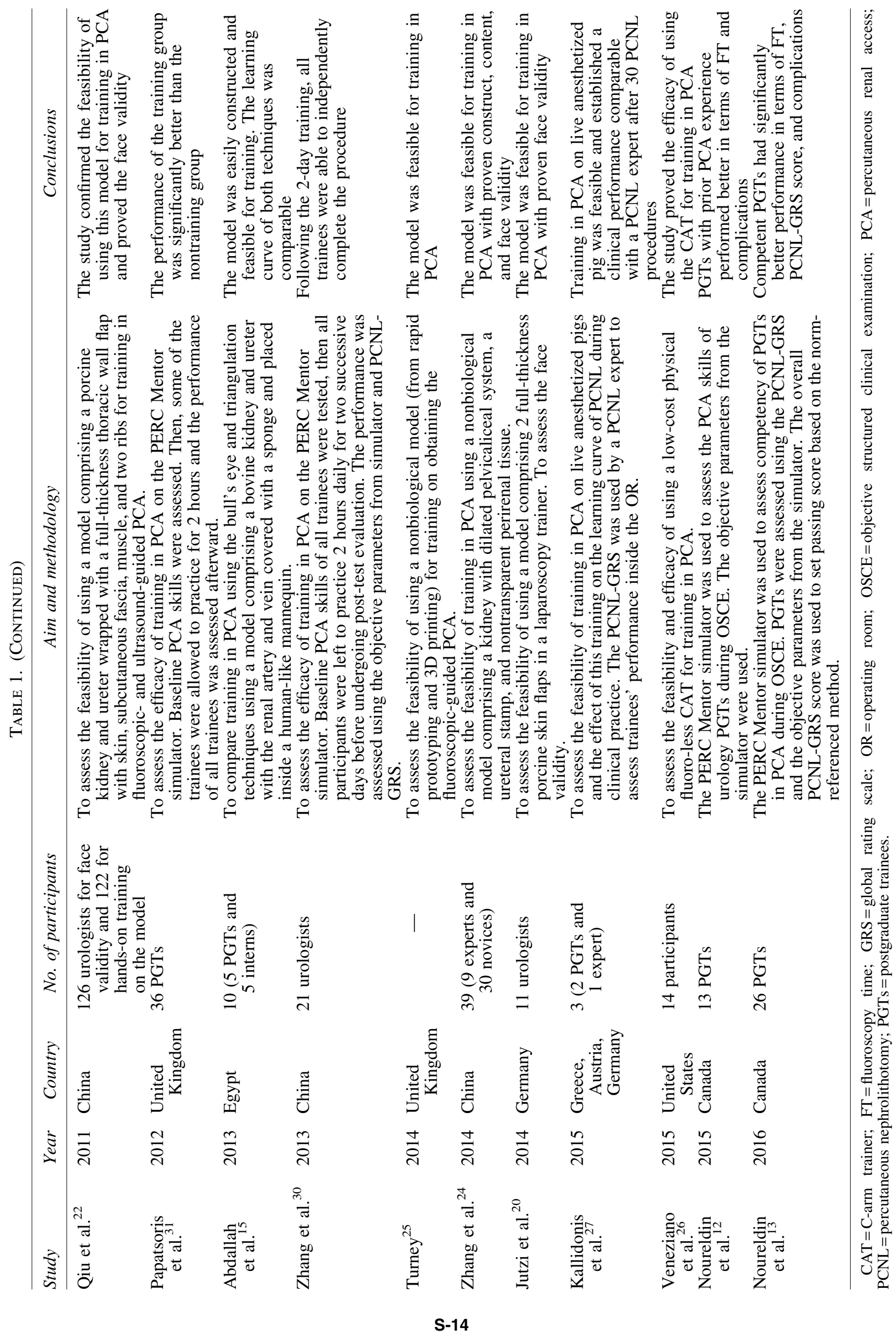




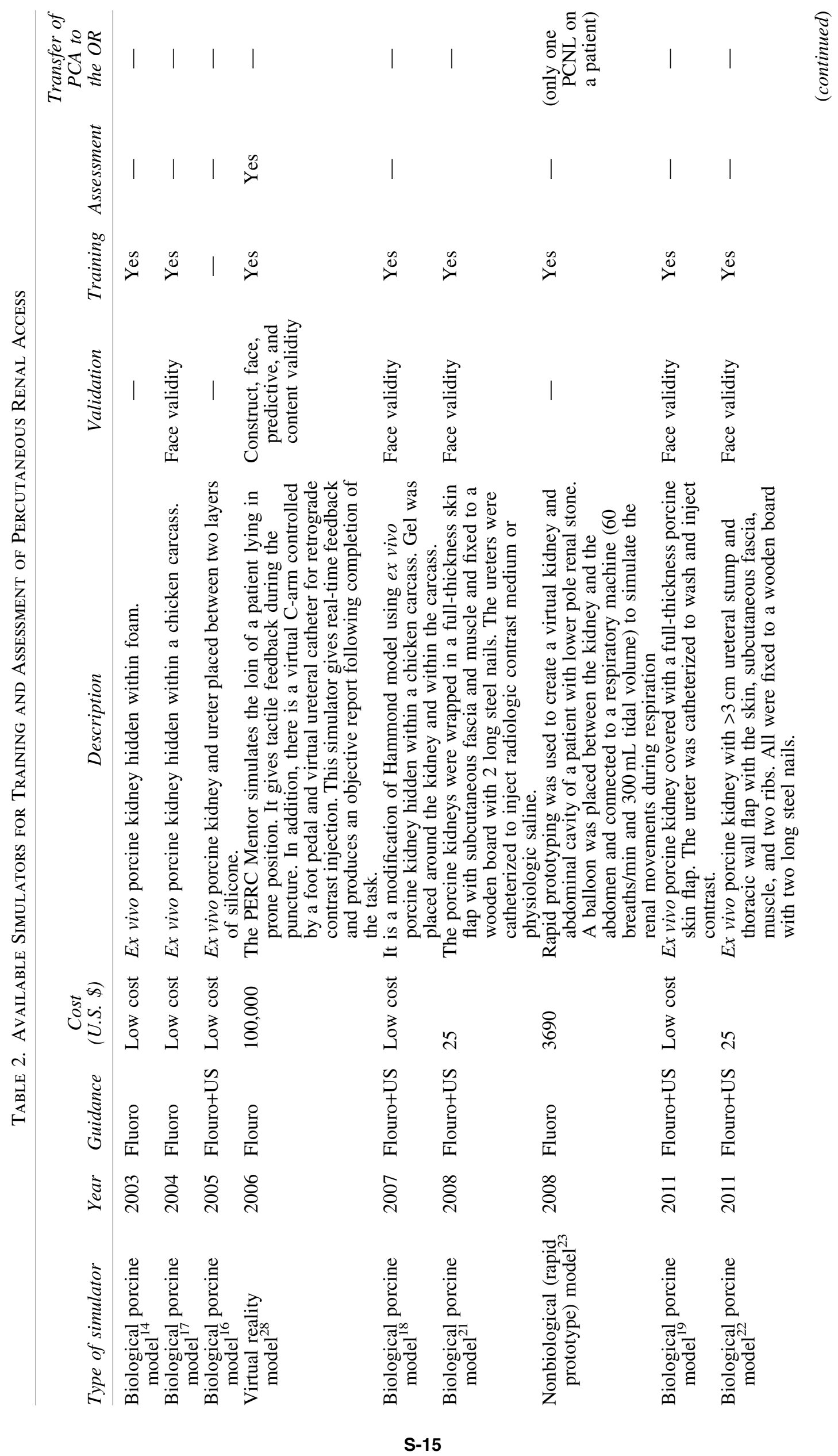




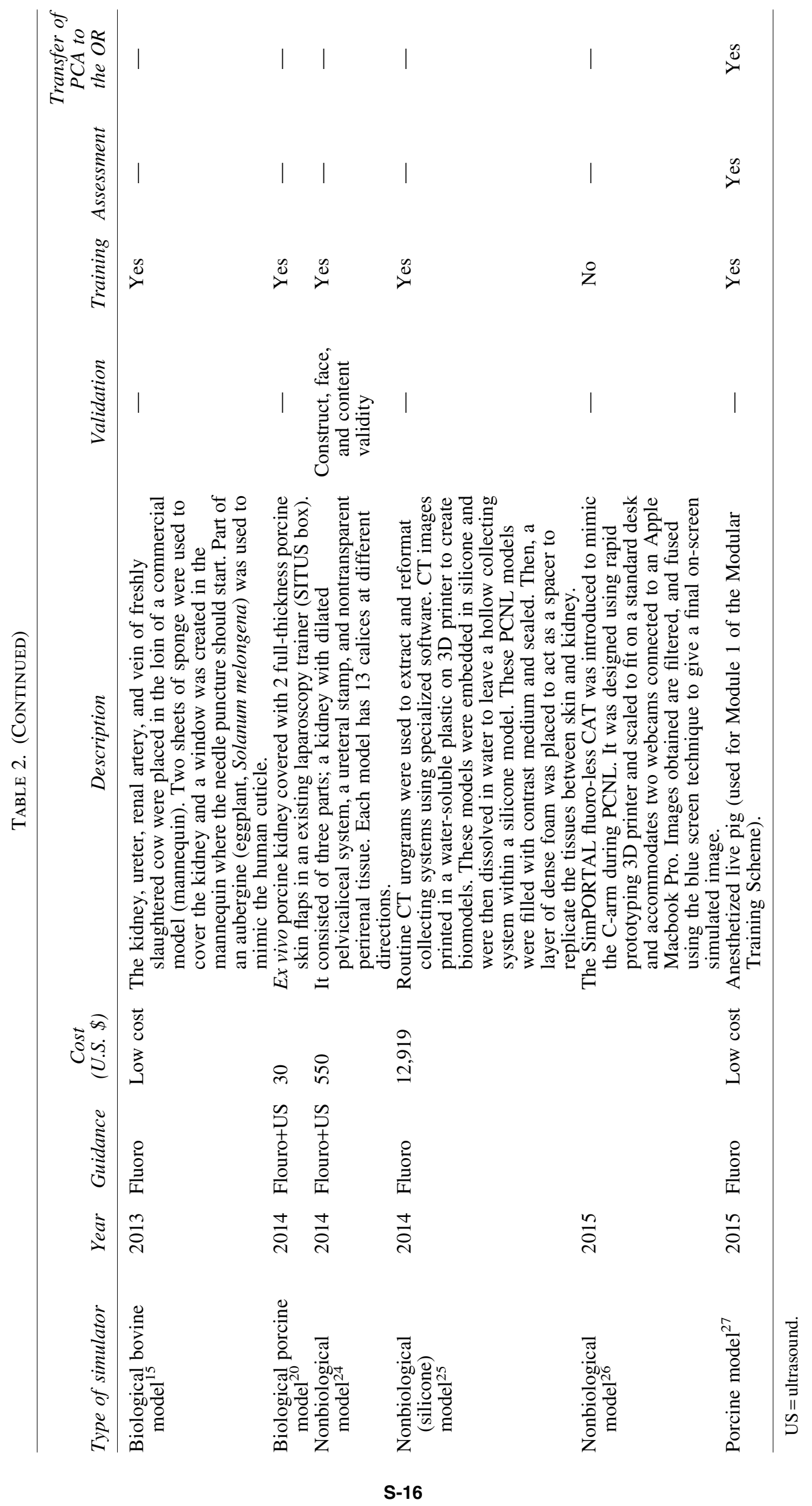


PERC Mentor simulator does not give a global score, the authors were not able to set a passing score for competency. Therefore, they performed another study to assess competency in PCA using task 4 on the PERC Mentor simulator, where each participant was asked to correctly access and pop seven balloons in seven renal calices. The 26 participants included PGTs from all four training programs in the province of Quebec from PGY-3 to 5. All participants were assessed both objectively using the PERC Mentor simulator and subjectively using the PCNL-GRS, and the competency passing score was calculated from the PCNL-GRS score using the norm-referenced method. Competent PGTs performed task 4 with significantly higher percentage of successful attempts to pop the balloons $(p<0.001)$, shorter FT (9.8 vs 6.5 minutes; $p=0.01)$, lower complications $(p=0.01)$, and higher PCNL-GRS scores $(p<0.001) .{ }^{13}$

\section{Transfer of PCA skills from VR to OR}

Most of the literature on VR simulation is related to laparoscopic surgery and gastrointestinal endoscopy. ${ }^{3,58}$ The analysis of clinical trials in a Cochrane review study reported the efficacy of training on VR simulators for acquisition of laparoscopic skills, ${ }^{59}$ However, there is paucity of data on the efficacy of training on VR simulators for endourologic skills, including PCNL. While three studies showed the potential improvement of performance following training on the PERC Mentor simulator, ${ }^{28,29,31}$ there is still lack of literature on the educational impact of training on simulators in terms of improved performance in PCA skills inside the OR. In a level 1 evidence-based study by Chou and co-workers, ${ }^{60}$ the early clinical practice of rigid URS improved following training on a VR simulator. Another study by Aloosh et al. recruited urology PGTs for training on the URO Mentor ${ }^{\mathrm{TM}}$ simulator (Simbionix). Trainees were assessed on the simulator and inside the OR using the validated URS-GRS. A significant positive correlation between URS-GRS scores obtained on the simulator and in the OR $(r=0.9, p=0.03)$ was found. Therefore, they concluded that the flexible URS skills obtained from training on the URO Mentor ${ }^{\mathrm{TM}}$ simulator could be transferred to the OR. ${ }^{61}$ However, there are no data on the transfer of PCA skills from training on VR simulators to the OR. Therefore, future prospective studies need to examine transfer of PCA skills from training on VR simulators to performance in the OR in terms of FT, OR time, and any complications relating to PCA.

\section{Conclusions}

There is paucity of literature regarding the current simulators for training and assessing PCA skills. The PERC Mentor simulator is currently the only validated VR simulator used for training and assessing PCA skills. However, it is expensive and there is no evidence regarding the transfer of PCA skills from the PERC Mentor to the OR. Therefore, more research is needed to validate these simulators and assess their educational impact for urology trainees and attending urologists.

\section{Acknowledgments}

This work was partially sponsored by Fonds de la Recherche Santé du Québec (FRSQ) Chercheur Boursier grant to Dr. Sero Andonian and by a grant from the Urology Care Foundation Research Scholars Program and the Boston Scientific Corporation, The Endourological Society, and the "Friends of Joe" to Dr. Yasser Noureldin and the CUASFSIU International Scholarship grant to Dr. Yasser Noureldin.

\section{Author Disclosure Statement}

No competing financial interests exist.

\section{References}

1. Stern J, Zeltser IS, Pearle MS. Percutaneous renal access simulators. J Endourol 2007;21:270-273.

2. Ng CF. Training in percutaneous nephrolithotomy: The learning curve and options. Arab J Urol 2014;12:54-57.

3. Watterson JD, Soon S, Jana K. Access related complications during percutaneous nephrolithotomy: Urology versus radiology at a single academic institution. J Urol 2006;176: 142-145.

4. Kauer PC, Laguna MP, Alivizatos G, et al.; on behalf of the members of ESUT. Present practice and treatment strategies in endourological stone management: Results of a survey of the European Society of Uro-technology (ESUT). Eur Urol 2005;48:182-188.

5. Lee CL, Anderson JK, Monga M. Residency training in percutaneous renal access: Does it affect urological practice? J Urol 2004;171:592-595.

6. Ghani KR, Andonian S, Bultitude M, Desai M, Giusti G, Okhunov Z, Preminger GM, de la Rosette J. Percutaneous nephrolithotomy: Update, trends, and future directions. Eur Urol 2016;70:382-396.

7. Ziaee SAM, Sichani MM, Kashi AH, et al. Evaluation of the learning curve for percutaneous nephrolithotomy. Urol J 2010;7:226-231.

8. Jang WS, Choi KH, Yang SC, et al. The learning curve for flank percutaneous nephrolithotomy for kidney calculi: A single surgeon's experience. Korean J Urol 2011;52:284288.

9. Noureldin YA, Elkoushy MA, Andonian S. Does the presence of a percutaneous renal access influence fluoroscopy time during percutaneous nephrolithotomy? Asian J Urol 2015;2:220-223.

10. Matsumoto ED, Hamstra SJ, Radomski SB, Cusimano MD. A novel approach to endourological training: Training at the Surgical Skills Center. J Urol 2001;166:1261-1266.

11. Mishra S, Jagtap J, Sabnis RB, Desai MR. Training in percutaneous nephrolithotomy. Curr Opin Urol 2013;23: 147-151.

12. Noureldin YA, Elkoushy MA, Andonian S. Assessment of percutaneous renal access skills during urology objective structured clinical examinations. Can Urol Assoc J 2015;9: 104-108.

13. Noureldin YA, Fahmy N, Anidjar M, Andonian S. Is there a place for virtual reality simulators in assessment of competency in percutaneous renal access? World J Urol 2016; 34:733-739.

14. Earp PP. Percutaneous renal surgery: New model for learning and training. Int Braz J Urol 2003;29:151-154.

15. Abdallah MM, Salem SM, Badreldin MR, Gamaleldin AA. The use of a biological model for comparing two techniques of fluoroscopy-guided percutaneous puncture: A randomised cross-over study. Arab J Urol 2013;11:79-84.

16. Strohmaier WL, Giese A. Ex vivo training model for percutaneous renal surgery. Urol Res 2005;33:191-193. 
17. Hammond L, Ketchum J, Schwartz BF. A new approach to urology training: A laboratory model for percutaneous nephrolithotomy. J Urol 2004;172(5 Pt 1):1950-1952.

18. Häcker A, Wendt-Nordahl G, Honeck P, Michel MS, Alken P, Knoll T. A biological model to teach percutaneous nephrolithotomy technique with ultrasound- and fluoroscopyguided access. J Endourol 2007;21:545-550.

19. Imkamp F, von Klot C, Nagele U, Herrmann TR. New exvivo organ model for percutaneous renal surgery. Int Braz $\mathrm{J}$ Urol 2011;37:388-394.

20. Jutzi S, Imkamp F, Kuczyk MA, Walcher U, Nagele U, Herrmann TR. New ex vivo organ model for percutaneous renal surgery using a laparoendoscopic training box: The sandwich model. World J Urol 2014;32:783-789.

21. Zhang Y, Ou TW, Jia JG, Gao W, Cui X, Wu JT, Wang G. Novel biologic model for percutaneous renal surgery learning and training in the laboratory. Urology 2008;72: 513-516.

22. Qiu Z, Yang Y, Zhang Y, Sun YC. Modified biological training model for percutaneous renal surgery with ultrasound and fluroscopy guidance. Chin Med J (Engl) 2011; 124:1286-1289.

23. Bruyère F, Leroux C, Brunereau L, Lermusiaux P. Rapid prototyping model for percutaneous nephrolithotomy training. J Endourol 2008;22:91-96.

24. Zhang Y, Yu CF, Jin SH, Li NC, Na YQ. Validation of a novel non-biological bench model for the training of percutaneous renal access. Int Braz J Urol 2014;40: 87-92.

25. Turney BW. A new model with an anatomically accurate human renal collecting system for training in fluoroscopyguided percutaneous nephrolithotomy access. J Endourol 2014;28:360-363.

26. Veneziano D, Smith A, Reihsen T, Speich J, Sweet RM. The SimPORTAL fluoro-less C-arm trainer: An innovative device for percutaneous kidney access. J Endourol 2015;29: 240-245.

27. Kallidonis $\mathrm{P}$, Kyriazis I, Vasilas M, et al. Modular training for percutaneous nephrolithotripsy: The safe way to go. Arab J Urol 2015;13:270-276.

28. Knudsen BE, Matsumoto $\mathrm{ED}$, Chew $\mathrm{BH}$, et al. A randomized, controlled, prospective study validating the acquisition of percutaneous renal collecting system access skills using a computer based hybrid virtual reality surgical simulator: Phase I. J Urol 2006;176:2173-2178.

29. Mishra S, Kurien A, Patel R, Patil P, Ganpule A, Muthu V, Sabnis RB, Desai M. Validation of virtual reality simulation for percutaneous renal access training. J Endourol 2010;24:635-640.

30. Zhang Y, Yu CF, Liu JS, Wang G, Zhu H, Na YQ. Training for percutaneous renal access on a virtual reality simulator. Chin Med J (Engl) 2013;126:1528-1531.

31. Papatsoris AG, Shaikh T, Patel D, Bourdoumis A, Bach C, Buchholz N, Masood J, Junaid I. Use of a virtual reality simulator to improve percutaneous renal access skills: A prospective study in urology trainees. Urol Int 2012;89: 185-190.

32. Frank JR, Snell LS, Cate OT, Holmboe ES, Carraccio C, Swing S. Competency-based medical education: Theory to practice. Med Teach 2010;32:638-645.

33. Simpson JG, Furnace J, Crosby J, et al. The Scottish doctorlearning outcomes for the medical undergraduate in Scotland: A foundation for competent and reflective practitioners. Med Teach 2002;24:136-143.
34. Frank JR, Snell LS, Sherbino J, eds. Draft CanMEDS 2015 Physician Competency Framework-Series III. Ottawa: The Royal College of Physicians and Surgeons of Canada; 2014 September. Available at: www.royalcollege.ca/portal/page/ portal/rc/common/documents/canmeds/framework/canmeds 2015_framework_series_III_e.pdf (Last accessed on June 18, 2016).

35. Frank JR, Danoff D. The CanMEDS initiative: Implementing and outcomes-based framework of physician competencies. Med Teach 2007;29:642-647.

36. Armagan A, Tepeler A, Silay MS, Ersoz C, Akcay M, Akman T, et al. Micropercutaneous nephrolithotomy in the treatment of moderate-size renal calculi. $\mathrm{J}$ Endourol 2013;27:177-181.

37. Ganpule AP, Bhattu AS, Desai M. PCNL in the twenty-first century: Role of microperc, miniperc, and ultraminiperc. World J Urol 2015;33:235-240.

38. de la Rosette JJ, Laguna MP, Rassweiler JJ, Conort P. Training in percutaneous nephrolithotomy-A critical review. Eur Urol 2008;54:994-1001.

39. Ather $\mathrm{MH}, \mathrm{Ng} \mathrm{CF}$, Pourmand G, Osther PJ. Training the resident in percutaneous nephrolithotomy. Arab J Urol 2014; 12:49-53.

40. Gill JD, Stewart LF, George NJ, Eardley I. Operative experience of urological trainees in the UK. BJU Int 2012; 109:1296-1301.

41. Allen D, O'Brien T, Tiptaft R, Glass J. Defining the learning curve for percutaneous nephrolithotomy. J Endourol 2005;19:279-282.

42. Tanriverdi O, Boylu U, Kendirci M, Kadihasanoglu M, Horasanli K, Miroglu C. The learning curve in the training of percutaneous nephrolithotomy. Eur Urol 2007;52:206-212.

43. Noureldin YA, Elkoushy MA, Andonian S. Assessment of photoselective vaporization of the prostate skills during urology objective structured clinical examinations (OSCE). Can Urol Assoc J 2015;9:e61-e66.

44. Noureldin YA, Aloosh M, Andonian S. How to use virtualreality simulators to assess competency in basic endourologic and robotic skills? Videourology January 2016; DOI: 10.1089/vid.2016.0014.

45. Ahmed K, Jawad M, Dasgupta P, Darzi A, Athanasiou T, Khan MS. Assessment and maintenance of competence in urology. Nat Rev Urol 2010;7:403-413.

46. Aydin A, Ahmed K, Shafi AM, Khan MS, Dasgupta P. The role of simulation in urological training-A quantitative study of practice and opinions. Surgeon 2015; pii: S1479666X(15)00059-1.

47. Epstein RM, Hundert EM. Defining and assessing professional competence. JAMA 2002;287:226-235.

48. Prystowsky JB, Bordage G, Feinglass JM. Patient outcomes for segmental colon resection according to surgeon's training, certification, and experience. Surgery 2002;132: 663-670; discussion 670-672.

49. Ahmed K, Jawad M, Abboudi M, et al. Effectiveness of procedural simulation in urology: A systematic review. J Urol 2011;186:26-34.

50. Feldman LS, Hagarty SE, Ghitulescu G, et al. Relationship between objective assessment of technical skills and subjective in-training evaluations in surgical residents. J Am Coll Surg 2004;198:105-110.

51. Shah J, Darzi A. Surgical skills assessment: An ongoing debate. BJU Int 2001;88:655-660.

52. Shaharan S, Neary P. Evaluation of surgical training in the era of simulation. World J Gastrointest Endosc 2014;6:436-447. 
53. Martin JA, Regehr G, Reznick R, MacRae H, Murnaghan J, Hutchison C, Brown M. Objective structured assessment of technical skill (OSATS) for surgical residents. Br J Surg 1997;84:273-278.

54. Aggarwal R, Grantcharov T, Moorthy K, Milland T, Darzi A. Toward feasible, valid, and reliable video-based assessments of technical surgical skills in the operating room. Ann Surg 2008;247:372-379.

55. Beard JD, Choksy S, Khan S. Assessment of operative competence during carotid endarterectomy. Br J Surg 2007; 94:726-730.

56. Moorthy K, Munz Y, Sarker SK, Darzi A. Objective assessment of technical skills in surgery. BMJ 2003;327: 1032-1037.

57. Reznick RK, MacRae H. Teaching surgical skills changes in the wind. N Engl J Med 2006;355:2664-2669.

58. Felsher JJ, Olesevich M, Farres H, et al. Validation of a flexible endoscopy simulator. Am J Surg 2005;189: 497-500.

59. Gurusamy KS, Aggarwal R, Palanivelu L, et al. Virtual reality training for surgical trainees in laparoscopic surgery. Cochrane Database Syst Rev 2009;1:CD006575.

60. Chou DS, Abdelshehid C, Clayman RV, McDougall EM. Comparison of results of virtual-reality simulator and training model for basic ureteroscopy training. J Endourol 2006;20:266-271.

61. Aloosh M, Noureldin YA, Andonian S. Transfer of flexible ureteroscopic stone-extraction skill from a virtual reality simulator to the operating theatre: A pilot study. J Endourol 2016; In press. PMID: 27532227; DOI:10.1089/end.2016.0365
Address correspondence to: Sero Andonian, MD, MSc, FRCSC, FACS Division of Urology McGill University

1001 Boulevard Decarie, Suite D05.5331

Montréal H4A $3 \mathrm{J1}$

Québec

Canada

E-mail: sero.andonian@muhc.mcgill.ca

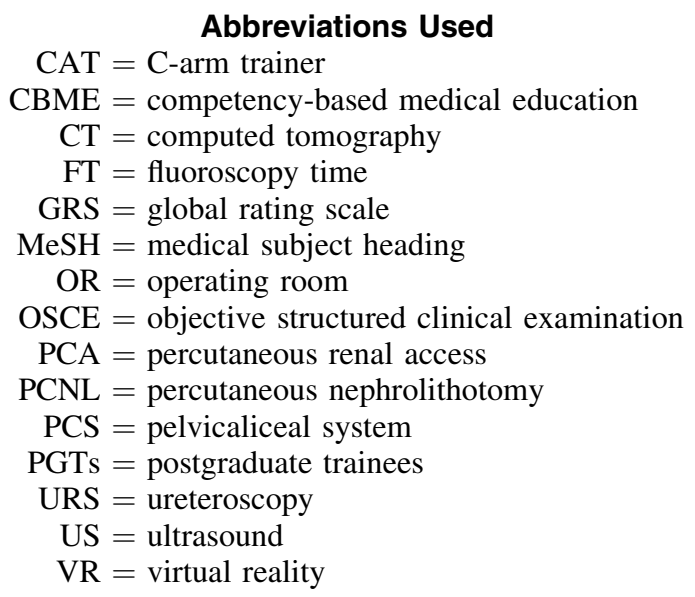

